\title{
Photopolymerization of Styrene in the Presence of Oxygen. Role of the Charge-Transfer Complex
}

\author{
Toshiyuki Kodarra, Kohji Hayashi, and Toshihito OHnishi \\ Department of Industrial Chemistry, Faculty of Engineering, \\ Fukui University, Fukui, Japan.
}

(Received January 11, 1972)

\begin{abstract}
Ultraviolet absorption spectra of several monomers such as styrene, $\alpha$-methylstyrene, isoprene, vinyl acetate, etc., were measured in nitrogen and oxygen atmospheres by bubbling the respective gases into the monomers at room temperature. The extra absorptions due to the oxygen which could be ascribed to contact chargetransfer complexes between monomers and oxygen with oxygen as an electron acceptor was found. The photopolymerization of styrene was carried out to investigate the role of the charge-transfer complex in it and was found to proceed several orders of magnitude faster in the presence of small amounts of oxygen than in its abscence. On the basis of polymer composition, viscosity measurement, the wavelength dependence of polymerization, and the ability of initial product to initiate polymerization, these facts could be explained as follows. Polystyrene peroxide is formed by photoirradiation of the charge-transfer complex between styrene and oxygen in the initial stage of the polymerization and the photoinduced decomposition of the polystyrene peroxide initiates the polymerization of the styrene. Some contribution from the exciplex between oxygen and excited singlet styrene to the formation of the alternating copolymer was also suggested on the basis of measurements of the fluorescence spectra of styrene.

KEY WORDS Vinyl Monomer / Styrene / Oxygen / Charge-Transfer

Complex / UV / Photopolymerization / Polystyrene Peroxide / Photodecomposition / Alternating Copolymer /
\end{abstract}

Some results of a study of photopolymerization of styrene (ST) were recently reported by the authors. ${ }^{1}$ It was noted that the presence of small amounts of $\mathrm{O}_{2}$ acceralates the photopolymerization of ST, as compared with polymerization in vacuo. The measurements of UV-absorption spectra revealed that extra absorption bands are caused by dissolved $\mathrm{O}_{2}$ which were attributed to contact charge-transfer complex between $\mathrm{ST}$ and $\mathrm{O}_{2}$, with oxygen as an electron acceptor. The importance of the charge-transfer complex between ST and $\mathrm{O}_{2}$ as a light absorbing species in the fundamental process of the photopolymerization of ST has been suggested. In the present paper the authors report some additional experimental results and discuss the detailed mechanism of the photopolymerization of ST in the presence of $\mathrm{O}_{2}$.

\section{EXPERIMENTAL}

\section{Materials}

Monomers were washed by the usual techniques and rectified through $60-\mathrm{cm}$ packed column three times under reduced nitrogen pressure just before use.

Commercially available oxygen was purified by passing through an aqueous solution saturated with potassium permanganate, a column packed with potassium hydroxide pellets, and concentrated sulfuric acid.

Commercial azobisisobutyronitrile (AIBN) was recrystallized twice from ethyl alcohol.

\section{Polymerization}

The monomers were sealed either in an air or an oxygen atmosphere after saturating them by bubbling the respective gas at $0^{\circ} \mathrm{C}$. The ampoules were placed horizontally in a water bath kept at $30^{\circ} \mathrm{C}$, and rotated on a radius of $5 \mathrm{~cm}$. The distance from the light to the axis 
of rotation was $17.5 \mathrm{~cm}$. The volume of ampoule was $20 \pm 1 \mathrm{~m} l$ and the amount of monomers was $6.2 \mathrm{~m} l$.

The copolymerizations of ST with $\mathrm{O}_{2}$ were carried out by bubbling $\mathrm{O}_{2}$ at the rate of 21 $\mathrm{m} l / \mathrm{min}$ through a capillary tube into ST loaded in a polymerization apparatus equipped with a reflux condenser protected from atmospheric moisture by calcium chloride. The apparatus was allowed to stand for the desired time in the water bath kept at $30^{\circ} \mathrm{C}$.

After a given time, the polymerization mixtures were poured into an excess of methyl alcohol. The precipitate was collected by filtration through a sintered glass funnel and dried at room temperature under reduced pressure. The polymers obtained in six hours were hard enough to be treated by precipitation, especially in the presence of $\mathrm{O}_{2}$. Therefore, ST was distilled off through a vacuum line without heating and the residue was freeze-dried from benzene solution. All the products obtained were stored in a refrigerator below $-10^{\circ} \mathrm{C}$. The vessels which contained monomer or product, were wrapped by aluminum foil to protect them from light during the operation except for the time under irradiation.

\section{Photoirradiation}

UV irradiation were carried out with a 100watt high pressure mercury lamp (Toshiba Electric Works, Model SHL-100UV-2). The control of wavelength was made either by the ampoule glass or by using Toshiba colored glass filters.

\section{Measurement of Spectra}

UV spectra were recorded by a Hitachi EPS3T Spectrophotometer and fluorescence spectra on a Model 204-Hitachi Fluorometer at room temperature respectively. The procedure used to measure UV and fluorescence spectra of monomers saturated with $\mathrm{O}_{2}, \mathrm{~N}_{2}$, or mixtures of them was essentially identical with that described by Tsubomura and Mulliken. ${ }^{2}$ The mixtures of $\mathrm{O}_{2}$ and $\mathrm{N}_{2}$ of desired composition were made in a $2-l$ four-necked round-bottomed flask equipped with a manometer, inlets to $\mathbf{O}_{2}$ and $\mathrm{N}_{2}$ from respective gas bombs and capillary outlets through which the gas mixtures were bubbled into the monomers.

\section{Viscosity Measurement}

Solution viscosity was determined in benzene at $30^{\circ} \mathrm{C}$ using an Ubbelohde viscometer. The intrinsic viscosity, $[\eta]$, was obtained from specific viscosities at four polymer concentrations.

\section{Determination of Carbonyl Compounds}

For the qualitative determination of carbonyl compounds formed during polymerization in the presence of $\mathrm{O}_{2}$, the filtrate obtained after collecting the precipitate in an excess of methanol was divided into two parts, A and B. $15 \mathrm{mg}$ of 2,4-dinitrophenylhydrazine in $5 \mathrm{ml}$ of $18-\%$ hydrochloric acid were added to the part $\mathrm{A}$ and allowed to stand overnight at $0^{\circ} \mathrm{C}$. The precipitate obtained was collected and its IR spectrum was compared with that of the authentic hydrazone of benzaldehyde. The part B was treated in a similar way but the hydrazones were extracted with benzene after addition of a saturated aqueous solution of sodium chloride until the benzene layer separated out. The extract was dried over Drierite and chromatographed on a thin layer of Silica-Layer G of Nakarai Chemicals Co. by ascending technique using an equivolume mixture of benzene and petroleum ether as the solvent system. Carbonyl compounds were identified by comparing $R_{\mathrm{f}}$ values with those of authentic samples.

\section{RESULTS AND DISCUSSION}

\section{Absorption Spectra}

UV absorption spectra of several monomers such as ST, isoprene(IP), $\alpha$-methylstyrene(MST), and vinyl acetate(VAc) were subtracted from those of the same samples saturated with $\mathrm{O}_{2}$. The resulting spectra for the absorption caused by $\mathrm{O}_{2}$ are shown in Figure 1. These absorption spectra were obtained reversibly by bubbling $\mathrm{O}_{2}$ and $\mathrm{N}_{2}$ alternatively through the substances for at least $1 \mathrm{~min}$. This is evidence that the extra absorption bands found are due to interactions between $\mathrm{O}_{2}$ and monomers, and not to the formation of oxidation products. In the cases of $\mathrm{ST}^{3}$ and $\mathrm{IP}^{4}$, it has already been reported that extra absorptions are observed, when their spectra are measured with $\mathrm{O}_{2}$ under high pressure. These results show that they are also observed in the presence of $\mathrm{O}_{2}$ under atmospheric pressure. For all cases, the wavelength 


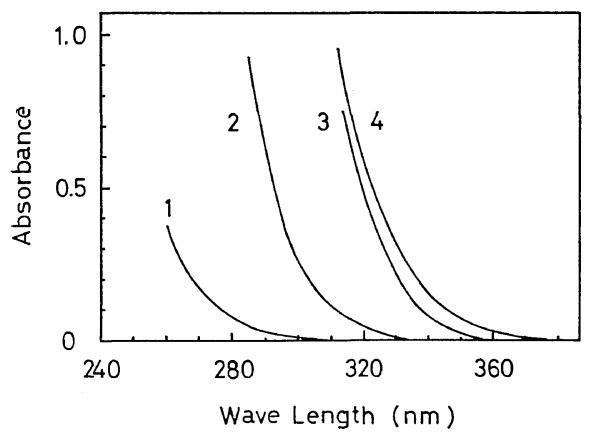

Figure 1. Ultraviolet absorption spectra caused by monomers (neat) saturated with $1 \mathrm{~atm}$ of oxygen (1-cm path length): 1, VAc; 2, IP; 3, ST; 4, MST.

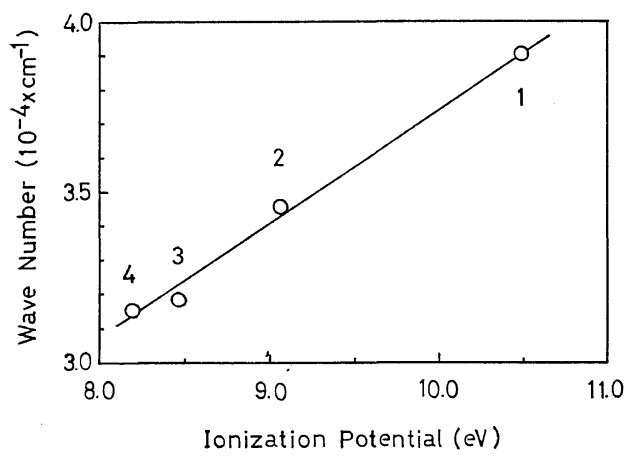

Figure 2. Relation between ionization potentials and absorption spectra: 1, VAc; 2, IP: 3, ST; 4, MST.

region for the absorption measurements was limited by the strong bands of monomers themselves and the absorption maxima of the spectra due to the interaction between $\mathrm{O}_{2}$ and monomers could not be observed. In order to obtain information on these absorption spectra, the wavenumbers at which the absorbance reaches the value of 0.7 were plotted against the ionization potentials ${ }^{5,6}$ in Figure 2. Since the absorbance of vinyl acetate did not attain the value of 0.7 in the experimental conditions, the wavenumber for $255 \mathrm{~nm}$ was adopted. Although corrections for the solubility of $\mathrm{O}_{2}$ in these monomers were not made, the plot gives a reasonably straight line. Plots of the absorbances $v s$. the partial pressure of $\mathrm{O}_{2}$ in equilibrium are illustrated for ST in Figure 3. It shows that linear relationship between them exist for every wavelength. On the basis of

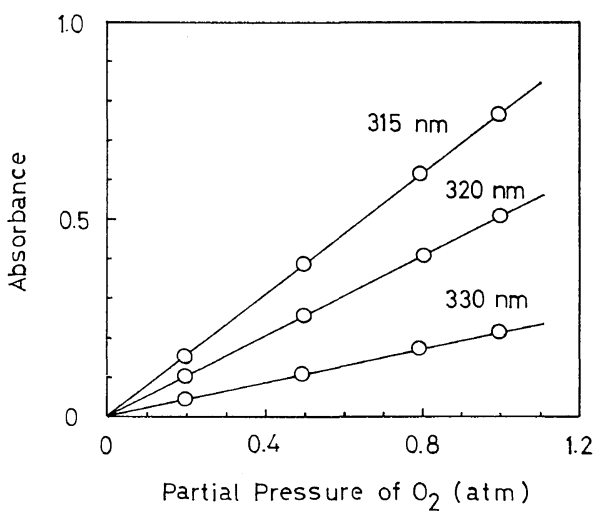

Figure 3. Plot of ultraviolet absorption intensity of ST vs. oxygen pressure.

these results, the extra absorption bands obtained by dissolved $\mathrm{O}_{2}$ are thought to be due to the charge-transfer transition. Similar absorptions observed for hydrocarbons, ${ }^{2,7-9}$ ethers, ${ }^{7,10}$ amines, ${ }^{2,11}$ and alcohols ${ }^{2,7}$ have been identified as due to the charge-transfer complexes ${ }^{2}$ of these compounds with $\mathrm{O}_{2}$.

On the other hand, both the unperturbed ${ }^{12}$ and perturbed ${ }^{4} \mathrm{~S} \rightarrow \mathrm{T}$ transition have been reported for olefins and aromatic compounds at almost the same wavelength as those of extra absorption bands which have been ascribed to charge-transfer transition. The assignment of the extra absorption bands to these transition is less probable, because the $\mathrm{S} \rightarrow \mathrm{T}$ transitions of ST and IP have been observed around 463 $\mathrm{nm}^{3}$ and $380 \mathrm{~nm}^{4}$ respectively. In addition, the continuous increase of absorption intensity with decreasing wavelength cannot be accounted for by $\mathrm{S} \rightarrow \mathrm{T}$ transition. ${ }^{8}$

Three other monomers have been subjected to the measurement of UV-absorption spectra in $\mathrm{O}_{2}$ and $\mathrm{N}_{2}$ atmosphere. They are 2-vinyl pyridine(VP), methyl methacrylate(MMA), and acrylonitrile(AN). It was found that they did not give extra absorption bands, which could be ascribed to the charge-transfer transition, although very small shifts which changed irreversibly on alternative bubbling of $\mathrm{O}_{2}$ and $\mathrm{N}_{2}$ were observed. In the case of MMA and VP, these facts might be explained by considering that the charge-transfer absorption overlaps with the strong absorption of the monomers them- 
selves, as discussed by Tsubomura and Mulliken for pyridine. $^{2}$ Although the ionization potentials of MMA and VP are not known, they are considered to be not less than $10.0 \mathrm{eV}$ for MMA and $8.0 \mathrm{eV}$ for VP, judging from the relation between the ionization potentials and the highest occupied $\pi$-orbital energies of several monomers, ${ }^{6}$ and the ionization potentials of related compounds, e.g., methyl acrylate, 10.7 $\mathrm{eV}^{6}$ and pyridine, $9.13 \mathrm{eV}^{2}$ From the values $(10.0 \mathrm{eV}$ for MMA and $8.0 \mathrm{eV}$ for $\mathrm{VP}$ ) and the linear relation in Figure 2, the absorptions due to the charge-transfer transition are considered to be those observed at $266 \mathrm{~nm}$ for MMA and at $325 \mathrm{~nm}$ for VP. These are indeed in the region of strong absorption by the respective monomers. The charge-transfer bands are expected to occur at around $250 \mathrm{~nm}$ for $\mathrm{AN}$ applying its ionization potential $\left(10.75 \mathrm{eV}^{6}\right)$ to the linear relation in Figure 2. Experimental results showed in this region only a slight increase in absorbance which changed irreversibly.

\section{Effect of Added Oxygen on the Photopolymeriza-} tion of $S T$

In order to study the role of the absorption caused by the interaction between $\mathrm{ST}$ and $\mathrm{O}_{2}$ on the photopolymerization of ST, polymerizations under various conditions were carried out. The transmittance of the Toshiba colored glass filters and the ampoule glasses used, and the absorbance of ST saturated with $\mathrm{O}_{2}$ and $\mathrm{N}_{2}$ are shown in Figure 4. The results obtained by the polymerization are shown in Figure 5. The photopolymerization of ST proceeds much more

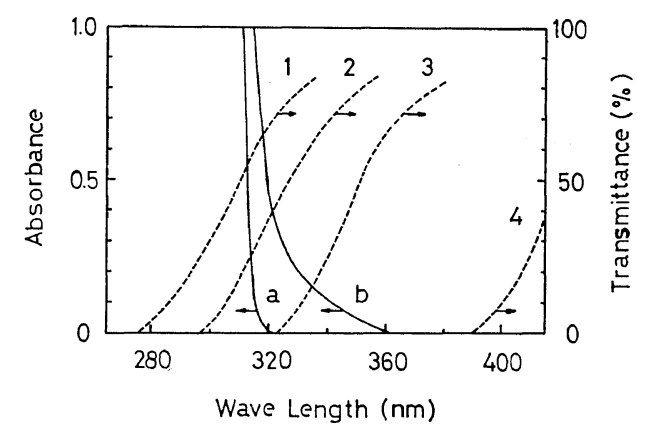

Figure 4. Ultraviolet-absorption spectra (1-cm path length) of ST (neat): a, ST in nitrogen atmosphere; b, ST in oxygen atmosphere. Dotted curves show the transmittance of ampoule glass and Toshiba colored glass filters: 1, IWAKI Code 7740 Glass (pyrex); 2, hard glass; 3, U-V 35 filter; 4, V-Y 42 filter.

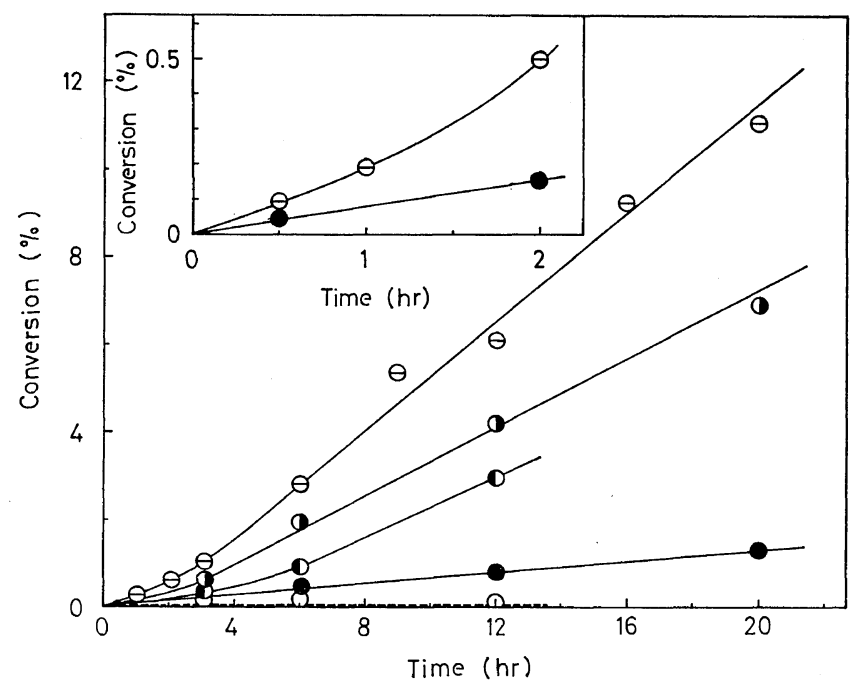

Figure 5. Conversion plotted against time for bulk polymerization of ST by UV irradiation in hard glass ampoule at $30^{\circ} \mathrm{C}$ : distance between light and polymerization tube, $17.5 \mathrm{~cm} ; \Theta, \mathrm{O}_{2} ; \boldsymbol{O}$, air; $-\mathrm{U}-\mathrm{V} 35, \mathrm{O}_{2} ; \bullet$, vacuo; $\bigcirc, \mathrm{O}_{2}$, [benzoquinone], $1.6 \times 10^{-2} \mathrm{~mol} / \mathrm{l}$. The initial stage of the polymerization in $\mathrm{O}_{2}$ and in vacuo is depicted in enlarged scale in the inset with the same notation as mentioned above. 
rapidly in the presence of small amounts of oxygen than in vacuo. Polymerization in the dark or using a V-Y 42 filter gives a conversion of $0.02 \%$ or $0.17 \%$ respectively after $12 \mathrm{hr}$. These results indicate that the light energy of shorter wavelength than $390 \mathrm{~nm}$ is mostly responsible for the rapid polymerization of ST with oxygen added. Considering the fact that the polymerization is inhibited by benzoquinone, polymerizations are thought to proceed by a radical mechanism as suggested in the previous paper. $^{1}$ In fact, Tsuji, et al., ${ }^{13}$ reported that there exists a charge-transfer complex between polyethylene and $\mathrm{O}_{2}$ and that $\mathrm{UV}$ irradiation of polyethylene in $\mathrm{O}_{2}$ gives greater radical yields than in $\mathrm{N}_{2}$. There seems to be an induction period in the polymerization with oxygen added. The polymers obtained in this period were hard to collect by precipitation in methanol and had to be treated as described in the experimental section.

The polymers obtained in hard glass ampoules in the presence of small amounts of oxygen by photoirradiation were subjected to elemental

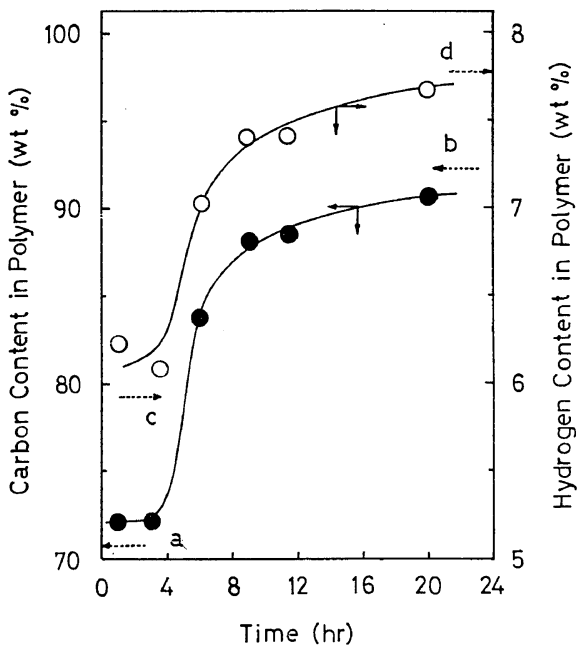

Figure 6. Time dependence of carbon and hydrogen contents in polymers obtained by the photopolymerization of ST with oxygen added in a hard glass ampoule at $30^{\circ} \mathrm{C}$ : $\bigcirc$, hydrogen content; -, carbon content. Dotted arrows show their calculated values; $a$ and $b$, carbon contents for PSPO and polystyrene respectively; $c$ and $d$, hydrogen contents for PSPO and polystyrene respectively.

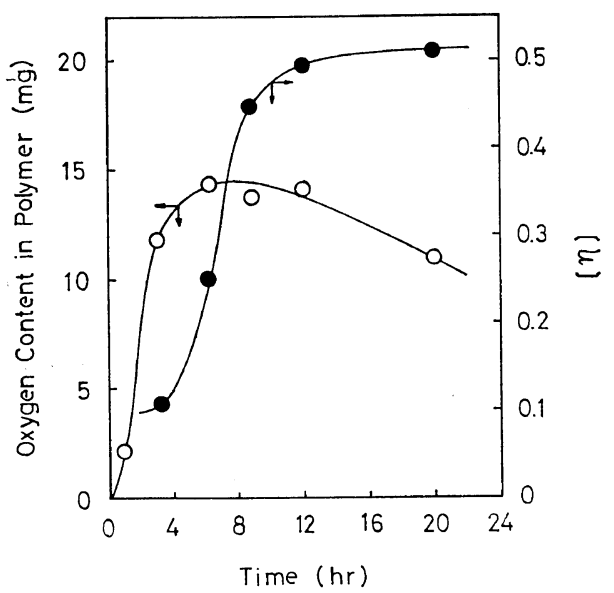

Figure 7. Time dependence of limiting viscosity, $[\eta]$, and oxygen content incorporated in polymers obtained in a hard glass ampoule with oxygen added at $30^{\circ} \mathrm{C}: \bullet,[\eta] ; \bigcirc$, oxygen content.

analyses and viscosity measurements. The carbon and hydrogen contents in polymers are plotted against polymerization time in Figure 6. The compositions of polymers obtained in the initial stage of polymerization (during the induction period) are almost the same as those of alternating copolymers of ST with $\mathrm{O}_{2}$, and they approach the composition of polystyrene, as the polymerization time increases. The amounts of oxygen incorporated in the polymers were calculated by subtracting carbon and hydrogen contents from the total weight of a given polymer. The results given in Figure 7 show that they increase rapidly with conversion and then decrease gradually without attaining the value of added oxygen (about $20 \mathrm{mg}$ ), while conversion continues to increase. The relationship between polymerization time and the limiting viscosity is also illustrated in Figure 7 for the same samples. They increase with polymerization time and then approach a constant value. The time dependence of limiting viscosity and carbon or hydrogen content shows good correspondence with each other. The limiting viscosity of polymers obtained by the photopolymerization for $12 \mathrm{hr}$ was $2.38 .^{1}$

The infrared spectra of polymers were measured in film prepared from chloroform solution on $\mathrm{NaCl}$ plate and are shown in Figure 8. The characteristic features of these 


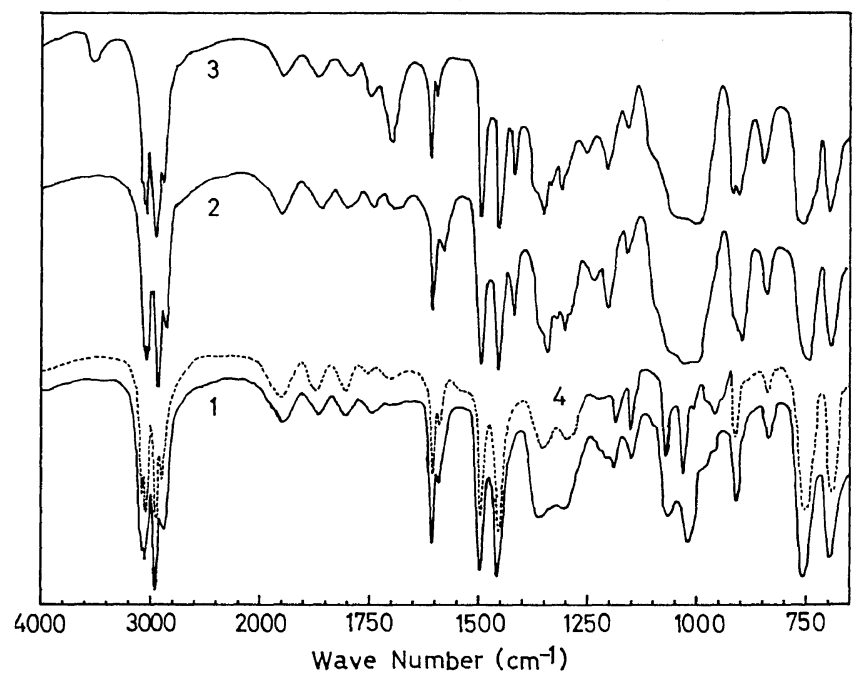

Figure 8. IR spectra of polymers derived from photopolymerization of ST under various conditions in a hard glass ampoule at $30^{\circ} \mathrm{C}$, measured in film prepared from chloroform solution on $\mathrm{NaCl}$ plate: $1, \mathrm{O}_{2}$, time, $12 \mathrm{hr}$; 2, $\mathrm{O}_{2}$, time, $3 \mathrm{hr} ; 3$, polymer obtained from copolymerization of ST with $\mathrm{O}_{2}$, time, $3 \mathrm{hr} ; 4$, standard polystyrene.

spectra, as compared with those of polystyrene, are that the polymer obtained from a short polymerization time ( 2 in Figure 8 ) indicates strong absorption due to a $\mathrm{C}-\mathrm{O}-\mathrm{O}-\mathrm{C}$ stretching vibration in the region between 1000 and $1100 \mathrm{~cm}^{-1} \cdot{ }^{14}$ As the polymerization time increases, the absorption caused by the peroxide bond becomes weaker, and the spectra approach the spectrum of polystyrene ( 1 and 4 in Figure 8). These results suggest that the alternating copolymers of ST with $\mathrm{O}_{2}$ (polystyrene peroxide: PSPO) are formed in the initial stage of the photopolymerization of ST in the presence of small amounts of $\mathrm{O}_{2}$. Alternating copolymer of ST with oxygen was obtained according to the known method, ${ }^{15}$ and its infrared spectrum agreed well with that of 2 in Figure 8 .

Photoirradiation of PSPO induces its decomposition to produce benzaldehyde and formaldehyde. $^{16}$ The filtrate obtained by collecting the precipitated polymer (polymerization condition, $9 \mathrm{hr}$ with oxygen added) was treated with 2,4-dinitrophenylhydrazine and its hydrazones of benzaldehyde and formaldehyde were confirmed by comparing thin layer chromatographs and IR spectra with those of authentic samples. It can be explained by these results that the quantity of $\mathrm{O}_{2}$ incorporated in polymers does not reach the amounts of added oxygen, and it decreases after attaining a maximum. The time when the polymer composition deviates from that of PSPO just corresponds to the end of induction period in Figure 5. These facts suggest that the PSPO formed in the initial stage of the polymerization is decomposed by photoirradiation to initiate the polymerization of the ST. To confirm this hypothesis, polymerizations of ST were carried out as shown in Table I. No. 3 in Table I indicates that PSPO has the ability to initiate polymerization of ST. Comparison of no. 3 with no. 4 in Table I shows that the contribution from the benzaldehyde or formaldehyde formed by decomposition of PSPO to the polymerization of ST is negligible. It is also shown that photoirradiation is necessary for the formation of PSPO (see no. 1 and 2 in Table I) and for its decomposition (see no. 6 and 7 in Table I).

The results mentioned above show that the alternating copolymerization of $\mathrm{ST}$ with $\mathrm{O}_{2}$ occurs with photoirradiation of the chargetransfer complex by using a U-V 35 filter, but it must be noticed that polymerization of ST in vacuo also proceeds when irradiated by the 
Table I. Bulk polymerization of $\mathrm{ST}^{\mathrm{a}}$

\begin{tabular}{|c|c|c|c|c|}
\hline \multirow[b]{2}{*}{ No. } & \multicolumn{2}{|c|}{$\mathrm{I}, \mathrm{O}_{2}$} & \multirow{2}{*}{$\frac{\text { II, Vacuo }}{\text { b }}$} & \multirow{2}{*}{$\underset{\%}{\text { Yield, }}$} \\
\hline & $\underset{\text { nm }}{\text { Wavelength, }}$ & $\underset{\mathrm{hr}}{\operatorname{Time}}$ & & \\
\hline 1 & $>295$ & 3 & $>325$ & 3.38 \\
\hline $2^{c}$ & Dark & 3 & $>325$ & 0.54 \\
\hline 3 & $>295$ & 3 & $>295$ & 9.00 \\
\hline $4^{d}$ & $>295$ & 3 & $>295$ & 8.30 \\
\hline 5 & $>295$ & 1 & $>325$ & 1.72 \\
\hline 6 & $>325$ & 3 & $>325$ & 2.56 \\
\hline 7 & $>325$ & 3 & Dark & 0.30 \\
\hline
\end{tabular}

a After polymerization under condition I, polymerization mixtures were evacuated by the usual freeze-thaw cycle and polymerized under condition II. Distance between light and polymerization ampoule, $17.5 \mathrm{~cm}$; temp, $30^{\circ} \mathrm{C}$.

b Time, $12 \mathrm{hr}$.

- Polymerization in vacuo under the same condition yields conversion of $0.33 \%$.

d After polymerization under condition I, ST was removed by distillation through vacuum line without heating and then by freeze-dry technique from benzene solution. To the residue was added freshly distilled ST to be polymerized under condition II.

Table II. Photo- and thermal-polymerization of ST by AIBN $^{\text {a }}$ in bulk

\begin{tabular}{cccccc}
\hline \multirow{2}{*}{$\begin{array}{c}\text { Time } \\
\text { hr }\end{array}$} & \multicolumn{2}{c}{ Photo at } & $30^{\circ} \mathrm{C}^{\mathrm{b}}$ & & \multicolumn{2}{c}{ Dark at } & $50^{\circ} \mathrm{C}$ \\
\cline { 2 - 3 } \cline { 5 - 6 } & $\mathrm{O}_{2}$ & Vacuo & & $\mathrm{O}_{2}$ & Vacuo \\
\hline 0.5 & 1.08 & 1.25 & & 1.25 & 1.37 \\
1.0 & 1.69 & 2.05 & & 2.05 & 2.30 \\
\hline
\end{tabular}

a $2.6 \times 10^{-2} \mathrm{~mol} / l$.

b Hard glass ampoules were used. Distance between light and polymerization ampoule, $17.5 \mathrm{~cm}$.

same light (see note $\mathrm{b}$ in Table I). Accordingly, it is possible to suggest that the mechanism of the formation of initiating species is identical both in vacuo and in the presence of $\mathrm{O}_{2}$. In order to know whether the UV light absorbed by charge-transfer complex participates in the fundamental process of PSPO formation or not, photo- and thermal-polymerizations of ST initiated by AIBN were undertaken in vacuo and in the presence of $\mathrm{O}_{2}$. The results are given in Table II. The presence of $\mathrm{O}_{2}$ retards both the photo- and thermal-polymerizations of
ST by AIBN as already reported, ${ }^{17}$ whereas the opposite is true for the polymerization in the absence of AIBN (as shown in the inset of Figure 5). It could therefore be concluded that the charge-transfer complex takes part in the formation of PSPO as a light-absorbing species. The role of the charge-transfer complex between organic compounds and oxygen in the photooxidation of them has already been investigated by several authors. ${ }^{8,10,18}$ The photopolymerization of ethylene is accelerated by the presence of small amounts of $\mathrm{O}_{2}$ and has been explained by the enhanced $\mathrm{S} \rightarrow \mathrm{T}$ transition of ethylene due to magnetic perturbation of $\mathrm{O}_{2}{ }^{19}$ Since the $\mathrm{S} \rightarrow \mathrm{T}$ transition for ST has been found at around $463 \mathrm{~nm},{ }^{3}$ direct excitation of ST to its triplet state and reaction from the state are less probable, considering the fact that light energy of shorter wavelength than $390 \mathrm{~nm}$ is effective for the reaction.

In contrast to the polymerization of ST, polymerization of MMA is inhibited by the presence of small amounts of $\mathrm{O}_{2}$ as shown in Figure 9, in spite of the fact that MMA copolymerizes with $\mathrm{O}_{2}$ to form alternating copolymer. ${ }^{20}$ As discussed above, the absorption due to charge-transfer complex between MMA and $\mathrm{O}_{2}$ are considered to appear at around $266 \mathrm{~nm}$, if it exists. The failure to polymerize MMA in the presence of small amounts of $\mathrm{O}_{2}$ might be

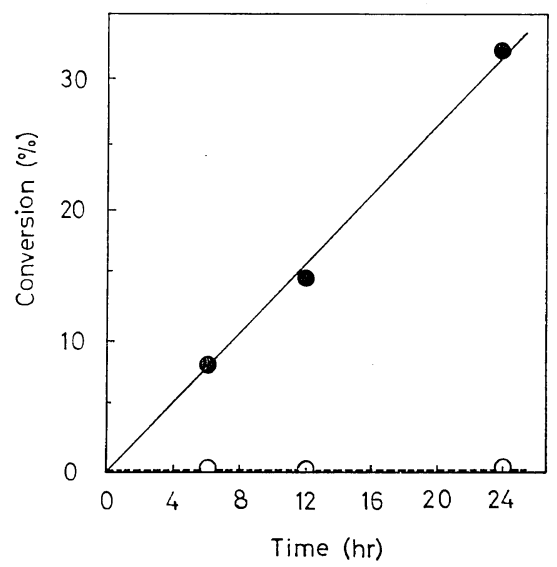

Figure 9. Conversion plotted against time for bulk polymerization of MMA in hard glass ampoule at $30^{\circ} \mathrm{C}$ : distance between light and polymerization ampoule, $17.5 \mathrm{~cm}$; $\bullet$ vacuo; $\bigcirc, \mathrm{O}_{2}$. 
ascribed to the fact that the light of longer wavelength $(>295 \mathrm{~nm}$ ) was used or to the very slow copolymerization of MMA with $\mathrm{O}_{2}$ compared with the homopolymerization of $\mathrm{MMA}^{15,21}$ Further study is, however, necessary to deduce a conclusion.

\section{Copolymerization of $\mathrm{ST}$ with $\mathrm{O}_{2}$}

Since it has been proved that the photoirradiation of the charge-transfer complex between ST and $\mathrm{O}_{2}$ induces alternating copolymerization of ST with $\mathrm{O}_{2}$, the wavelength dependence of copolymerization behavior was studied. The results in Figure 10 show that the copolymerization proceeds faster, as the wavelength of irradiated light becomes shorter. The copolymerization in the dark yielded a conversion of $0.01 \%$ after five hours. The fact that the copolymerization proceeds in almost the same way both in pyrex and hard glass ampoules after a certain initial period suggests that the polymerization is initiated mostly by the decomposition of PSPO after the time. The measurement of IR spectra also supports this assumption. The spectrum of polymer obtained by polymerization for one hour is the same as that of 2 in Figure 8, but the absorption due

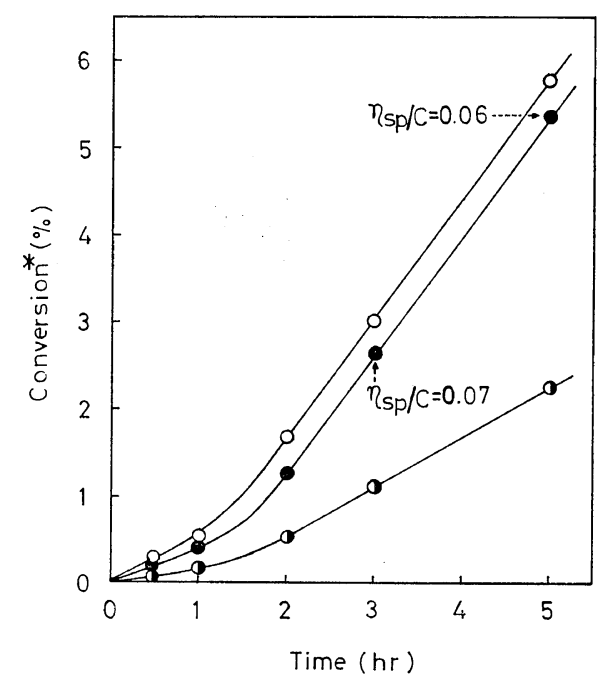

Figure 10. Plot of conversion $v s$. time for copolymerization of ST with $\mathrm{O}_{2}$ at $30^{\circ} \mathrm{C}: \mathrm{O}_{2}, 21 \mathrm{ml} / \mathrm{min}$; distance between light and polymerization apparatus, $10 \mathrm{~cm} ; \eta_{\mathrm{sp}} / \mathrm{C}, \mathrm{d} l / \mathrm{g} ; \bigcirc$, pyrex; $\odot$, hard glass; U-V 35 filter; *, based on ST.

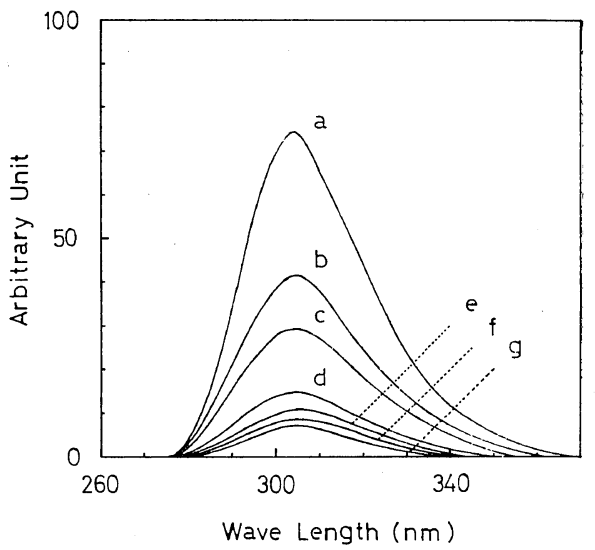

Figure 11. Fluorescence of ST $\left(2 \times 10^{-4} \mathrm{~mol} / l\right)$ in the presence of $\mathrm{O}_{2}$ : solvent, $n$-hexane; excitation, $265 \mathrm{~nm}$; partial pressure of $\mathrm{O}_{2}$ in the mixtures of $\mathrm{N}_{2}$ and $\mathrm{O}_{2}$ in atm, a, $0 ; \mathrm{b}, 0.10 ; \mathrm{c}, 0.21 ; \mathrm{d}, 0.46$; e, $0.70 ; \mathrm{f}, 0.85 ; \mathrm{g}, 1.00$.

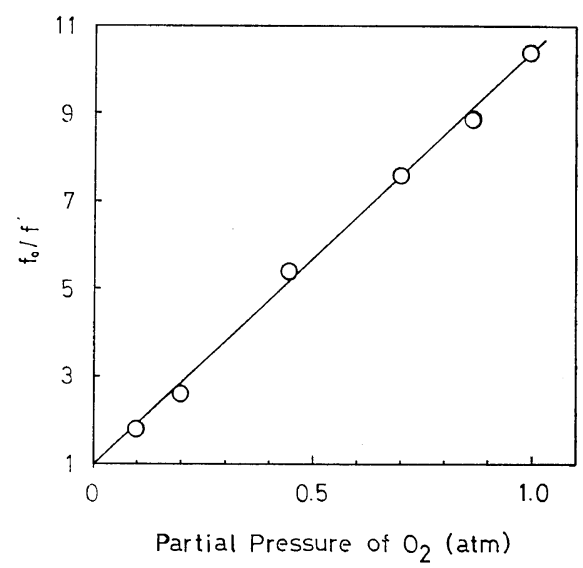

Figure 12. Stern-Volmer plot of $\mathrm{ST}-\mathrm{O}_{2}$ system: [ST], $2 \times 10^{-4} \mathrm{~mol} / l$, solvent, $n$-hexane; f at $305 \mathrm{~nm}$.

to streching vibration of $\mathrm{C}=\mathrm{O}$ double bond appears in the spectrum of the polymer obtained by polymerization for five hours.

To investigate the interaction of oxygen with the singlet excited state of ST, fluorescence spectra of ST were measured in the presence of $\mathrm{O}_{2}$ under various pressures. As shown in Figures 11 and 12, the fluorescence of ST is quenched by $\mathrm{O}_{2}$ according to Stern-Volmer plot. The quenching process of the fluorescence of aromatic compounds by $\mathrm{O}_{2}$ was investigated by several authors ${ }^{9,22,23}$ and it has been generally 
considered that it proceeds through the chargetransfer interaction between aromatic compounds and $\mathrm{O}_{2}{ }^{2}$ Therefore it is considered that photoinduced alternating copolymerization of ST with $\mathrm{O}_{2}$ proceeds through following two primary processes.

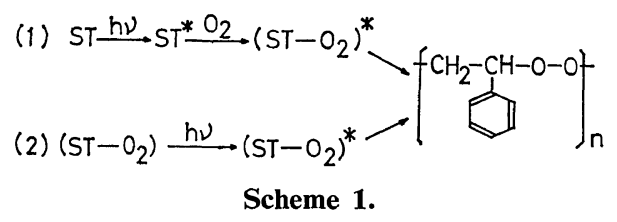

At present it is not clear whether the excited triplet charge-transfer complex produces PSPO directly or whether it transfers to other states which are responsible for the reaction.

\section{REFERENCES}

1. T. Kodaira and K. Hayashi, J. Polym. Sci., Part B, 9, 907 (1971).

2. H. Tsubomura and R.S. Mulliken, J. Amer. Chem. Soc., 82, 5966 (1960).

3. D. F. Evance, J. Chem. Soc., 1351, 3885 (1957).

4. D. F. Evance, ibid., 1736 (1960).

5. J.D. Morrison and A. J.C. Nicholson, $J$. Chem. Phys., 20, 1021 (1952).

6. T. Kagiya, "Kagaku Hannoh no Sokudoronteki Kenkyu-ho," Kagaku Dohjin Co., Ltd., Kyoto, 1970, p 814.
7. A. U. Munk and J. F. Scott, Nature, 177, 587 (1956).

8. J. C. W. Chien, J. Phys. Chem., 69, 4317 (1965).

9. H. Ishida, H. Takahashi, and H. Tsubomura, Bull. Chem. Soc. Japan, 43, 3130 (1970).

10. V. I. Stenberg, R. D. Olson, C. T. Wang, and N. Kulevsky, J. Org. Chem., 32, 3327 (1967).

11. H. Ishida, H. Takahashi, H. Sato, and H. Tsubomura, J. Amer. Chem. Soc., 92, 275 (1970).

12. C. Reid, J. Chem. Phys., 18, 1299 (1950).

13. K. Tsuji and T. Seiki, Polymer J., 2606 (1971).

14. L. J. Bellamy, "Infrared Spectra of Complex Molecules," Methuen and Co., Ltd., London, 1958, p 121.

15. R. Kerber and V. Serini, Makromol. Chem., 140, 1 (1970).

16. F. R. Mayo and A. A. Miller, J. Amer. Chem. Soc., 78, 1023 (1956).

17. F. R. Mayo, ibid., 80, 2465 (1958).

18. Hsueh-Chon Wu, Chin-Chang Chu, and TiChieh Ho, Hua Hsueh Hsueh Pao, 30, 241 (1964); Chem. Abstr., 61, 11489f (1964).

19. M. Hagiwara, H. Okamoto, T. Kagiya, and T. Kagiya, J. Polym. Sci., Part A-1, 8, 3295 (1970).

20. C. E. Barnes, R. M. Elofson, and G. D. Jones, J. Amer. Chem. Soc., 72, 210 (1950).

21. F. R. Mayo and A. A. Miller, ibid., 80, 2493 (1958).

22. W. R. Ware, J. Phys. Chem., 66, 455 (1962).

23. B. Stevens and B.E. Algar, ibid., 72, 2582 (1968). 